\title{
Scale Factor Duality and the Energy Condition Inequalities
}

\author{
Sayan Kar 6 \\ Inter University Centre for Astronomy and Astrophysics, \\ Post Bag 4, Ganeshkhind, Pune, 411 007, INDIA
}

\begin{abstract}
We demonstrate, by a simple analysis, that cosmological line elements related by scale factor duality also exhibit a duality with respect to the conservation/violation of the Weak Energy Condition (WEC) by the matter that acts as the source in the one-loop $\beta$ function equations for the metric coupling written explicitly in the form of the Einstein equations. Furthermore, a study of specific pairs of line elements (obtained via $\mathrm{O}(\mathrm{d}, \mathrm{d})$ transformations) hints at a possible generalisation of the above duality w.r.t. WEC for the case of $O(d, d)$ related spacetimes. Consequences and extensions thereof are also pointed out.
\end{abstract}

*Electronic Address : sayan@iucaa.ernet.in 
Scale factor duality as a symmetry of the string equations of motion in a curved background as well as low energy effective string actions was first discovered by Veneziano [1] in 1991. Shortly thereafter, it was extended to a more general symmetry of the effective action known as $O(d, d)$ [2] where $d$ denotes the number of coordinates of which the metric and matter fields are independent. Subsequent generalisations to background fields depending on more than one coordinate have been carried out in [4]. Such noncompact symmetries of string theory [5] have been exploited to a great extent to construct inequivalent string vacua and has yielded interesting background geometries representing black holes [6] and black p-branes [4], [7] as well as cosmologies [8], [9].

Stringy cosmologies (for reviews, see [8], [9] ) have certain characteristic features apart from the symmetry of scale factor duality. Firstly, one does not need to bring in an ad-hoc scalar field to get the necessary inflationary phase. The dilaton field which arises as one of the massless excitations of the string world sheet serves the purpose. Moreover, there exists the notion of a phase termed as pre-big-bang $(t<0)$ during which we have a rapidly inflating universe with a scale factor generically obeying a pole law $\left(a(t)=(-t)^{\beta} ;(\beta<0\right.$ ). This new phase is expected to end at $t=0$ where a FRW evolution $\left(a(t)=t^{\beta} ; 0<\beta<1\right)$ takes over and we finally end up with our universe today. Unfortunately, the transition from the pre-big-bang epoch to the FRW phase is plagued by the presence of a singularity, which, if absent would have solved the problem of singularities in GR, at least, in a cosmological setting. The generic presence of a singularity in the transition epoch which spoils a smooth crossover has been termed as the graceful exit problem in string cosmology [10]. Recently, Rey [11] has claimed that by introducing quantum back reaction it is possible to avoid the graceful exit problem at least within the limits of a two dimensional model like that of CGHS [12]. Whether an extension of this to four dimensions is possible or not is still an open question. On the other hand, a quantum cosmology approach to graceful exit has been advocated in [13].

The low energy effective theory that emerges out of string theory is much like Einstein gravity. The equations are derivable from an action which resembles that of a Brans-Dicke 
theory with the $\omega$ parameter set to -1 . Specifically, one has :

$$
S_{e f f}=\int d^{D} x \sqrt{-g} e^{-2 \phi}\left[V-R-4(\nabla \phi)^{2}+\frac{1}{12} H_{\mu \nu \alpha} H^{\mu \nu \alpha}\right]
$$

This is the bosonic sector of the genus-zero, low energy action for closed superstrings in the limit when inverse string tension (or $\alpha^{\prime}$ ) goes to zero. Here, $H_{\mu \nu \lambda}$ is the third-rank antisymmetric tensor field, $\phi$ the dilaton field and $V$ contains contributions from the dilaton potential and the cosmological constant. The $\beta$ function equations which are obtained by imposing quantum conformal invariance in the worldsheet sigma model can be derived from this action by performing appropriate variations.

Obviously, there are two frames in which the metrics look very different- the string (Brans-Dicke) and the Einstein frame. These frames are related to each other by a conformal transformation. We shall exclusively work in the string frame.

The Einstein-like equations can be written in the form $G_{\mu \nu}=e^{2 \phi} T_{\mu \nu}$ where $T_{\mu \nu}=$ $T_{\mu \nu}^{\phi}+T_{\mu \nu}^{M}(M$ denotes matter fields other than the dilaton). These equations are exactly those for Brans-Dicke theory with the parameter $\omega=-1$. One might argue that it is not proper to rewrite the $\beta$ function equations in an explicit Einstein form because at the level of the worldsheet sigma model $g_{\mu \nu}, \phi$ or $B_{\mu \nu}$ - all have the same status-they are the background fields in which strings propagate. We give here two reasons justifying our stand regarding rewriting the $\beta$ function equations as Einstein equations.

(i) If one has to compare and contrast low energy effective string models with GR then it is essential to rewrite the $\beta$ function equations in the form of a BD theory. There is nothing erroneous in this - no conservation laws are violated and everything is consistent. The $\phi$ and $B_{\mu \nu}$ fields as well as others act as sources for the metric. Moreover, it has been claimed that the probable violation of the WEC essentially invalidates the applicability of the singularity theorems of GR to the case of string inspired gravity [7]. This is ofcourse not entirely true as is shown for the case of black holes in [14] and for cosmologies in this paper.

(ii) At the level of pseudo - Riemannian geometry the Energy conditions can be thought of as conditions on the Ricci tensor of the geometry evaluated along a timeike/null geodesic, 
which ensures the occurence of geodesic focusing. Therefore, one can check the energy conditions (or, more precisely the null or timelike convergence condition) solely with the purpose of finding out whether geodesics can focus in a certain geometry. The presence of repulsive forces does actually emerge from this sort of an analysis.

We shall assume $T_{\mu \nu}^{M}=0$ (although there do exist many solutions with axionic, moduli, other matter fields as well as higher order terms in the Lagrangian [15]). Therefore we have only the dilaton field to worry about. The stress-energy for the dilaton field is given as :

$$
T_{\mu \nu}^{\phi}=e^{-2 \phi}\left[-2 \nabla_{\mu} \nabla_{\nu} \phi+g_{\mu \nu} \nabla^{2} \phi\right]
$$

One can therefore check the energy condition inequalities and conclude about the nature of the dilatonic matter that threads a stringy solution. This exercise has been recently done for the stringy black hole geometries [14]. We intend to do the same for stringy cosmologies in this paper. As demonstrated in a large part of the paper we find that scale-factor duality is directly related to the violation/conservation of the WEC. If a scale factor $a(t)$ is generated out of matter fields which satisfy the WEC then its dual $\tilde{a}(t)=\frac{1}{a(t)}$ can violate the $W E C$. Thus a pre-big-bang phase can be marked by a violation of the WEC whereas the FRW phase must necessarily satisfy it. This feature is irrespective of whether graceful exit happens or not. It should be mentioned that a duality in the values of the ADM masses (in the sense of opposite signs) of pairs of T-dual solutions of low energy effective string theory had been noted for special cases in [16. However, it was shown that there do exist counterexamples to this and therefore it is not a generic feature of $\mathrm{T}$-dual spacetimes [16]. Brief discussions on the violation/ conservation of the Energy Conditions and their role in avoiding the singularity theorems can be also found in [17], [18].

To begin, let us write down the line element for a homogeneous, isotropic cosmological model in $D$ spacetime dimensions. This is given as :

$$
d s^{2}=-d t^{2}+a^{2}(t)\left[\frac{d r^{2}}{1-k r^{2}}+r^{2} d \Omega_{D-2}^{2}\right]
$$

where $a(t)$ is the scale factor and the term in square brackets can be thought of as a metric on $S^{D-2}, R^{D-2}$ or $H^{D-2}$ (for $k=1,0,-1$ respectively). We shall work mostly with 
the $k=0$ case. From the metric in (1) we can write down the Einstein tensor $G_{\mu \nu}$ and hence equate it to the matter stress energy $e^{2 \phi} T_{\mu \nu}^{\phi}$. Defining a diagonal $T_{\mu \nu}^{\phi}$ (which in actuality is comprised out of the dilatonic matter) by the functions :

$$
e^{2 \phi} T_{00}^{\phi}=\rho(t) \quad ; \quad e^{2 \phi} T_{i i}^{\phi}=p(t)
$$

we can straightaway write down the Einstein equations and the Weak Energy Condition (WEC) inequalities. Before we do that let us recall the WEC .

Weak Energy Condition :

If $T_{\mu \nu}$ is the matter energy momentum tensor then the condition $T_{\mu \nu} \xi^{\mu} \xi^{\nu} \geq 0$ for all nonspacelike $\xi^{\mu}$ is known as the WEC. For a diagonal stress energy tensor $\left(T_{\mu \nu} \equiv \operatorname{diag}\left(\rho, p_{i}\right)\right)$ we have :

$$
\rho \geq 0 \quad ; \quad \rho+p_{i} \geq 0 \quad(i=1,2, \ldots . D-2)
$$

Physically, the WEC implies the fact that matter energy density has to be positive in all frames of reference. The $\rho+p_{i} \geq 0$ inequalities can be shown to be equivalent to the positivity of energy density in a suitably chosen frame of reference $\left(\rho^{\prime} \geq 0\right.$. The subset of conditions obtained by assuming $\xi^{\mu}$ to be null are known as the Null Energy Condition (NEC). This involves (for a diagonal $T_{\mu \nu}$ ) only the $\rho+p_{i} \geq 0$ inequalities. (In a geometric sense one can prefer calling these conditions on matter as those on geometry by referring to them as the timelike convergence and null convergence condition).

We therefore have :

(I) Einstein equations :

$$
\begin{array}{r}
\rho(t)=\left[\frac{(D-1)(D-2)}{2}\left\{\left(\frac{\dot{a}}{a}\right)^{2}+\frac{k}{a^{2}}\right\}\right] \\
p(t)=\left[\frac{(D-2)}{2}\left\{-(D-3)\left(\frac{\dot{a}}{a}\right)^{2}-\frac{\ddot{a}}{a}-\frac{(D-3) k}{a^{2}}\right\}\right]
\end{array}
$$

(II) WEC Inequalities :

$$
\begin{array}{r}
\rho(t)=\left[\frac{(D-1)(D-2)}{2}\left\{\left(\frac{\dot{a}}{a}\right)^{2}+\frac{k}{a^{2}}\right\}\right] \geq 0 \\
\rho(t)+p(t)=\left[(D-2)\left\{\left(\frac{\dot{a}}{a}\right)^{2}-\frac{\ddot{a}}{a}+\frac{k}{a^{2}}\right\}\right] \geq 0
\end{array}
$$


The Einstein equations for $k=0$ are invariant under the following set of transformations which comprise the notion of scale factor duality.

$$
\bar{a}=\frac{1}{a} \quad ; \quad \bar{\phi}=\phi-\operatorname{Tr}(\ln a)
$$

Note that by this $\rho, p$ go over to $\bar{\rho}$ and $\bar{p}$ respectively. The invariance of the Einstein equations comes from including the transformation for the $\phi$ field in the actual expressions for $\rho$ and $p$.

For $k=0$ one can see that a condition for the conservation of the WEC inequalities is given as :

$$
F(t)=\left(\frac{\dot{a}}{a}\right)^{2}-\frac{\ddot{a}}{a} \geq 0
$$

Now let us replace $a(t)$ by its dual scale factor $\bar{a}(t)=\frac{1}{a}$ Note now that the condition in terms of the quantity $\bar{a}$ turns out to be

$$
-\left(\frac{\dot{\bar{a}}}{\bar{a}}\right)^{2}+\frac{\ddot{\bar{a}}}{a} \geq 0
$$

which is exactly opposite to the condition for the original scale factor $a(t)$. Therefore, if $a(t)$ satisfies the WEC, then $\bar{a}(t)$ must necessarily violate it. Therefore, in choosing specific transformations to have invariance (of the Einstein equations) we have sacrificed the positivity of matter stress energy in any frame. The stress-energy for the field $\phi$ does conserve the WEC (for choices of $a$ ) but that for $\bar{\phi}$ does not. The opposite of this statement is also true for different choices of $a$.

One can also see this by looking at the explicit expressions for the WEC in terms of the scalar field stress energy. The WEC turns out to be given as :

$$
\begin{gathered}
\rho=-2 \dot{\phi}^{2}+6 \dot{\phi} \frac{\dot{a}}{a} \geq 0 \\
\rho+p=-2 \dot{\phi}^{2}+4 \dot{\phi} \frac{\dot{a}}{a} \geq 0
\end{gathered}
$$

Note that the first of these equations remain invariant under the scale factor duality transformations for the scale factor and the scalar field. The second one picks up an overall 
negative sign and is therefore consistent with the expression obtained by considering the R.H.S. of the Einstein equation.

Let us demonstrate this fact with a pair of dual scale factors that generically arise in string cosmology. We have

$$
a(t)=(t)^{\beta} \quad(0<\beta<1) \quad ; \quad \bar{a}(t)=(-t)^{\beta} \quad(\beta<0)
$$

Note that the domain of the dual scale factor is $t \leq 0$ while for the other one it is $t \geq 0$.

Evaluating the expression for $F(t)$ one can very easily see that the dual scale factor would violate the WEC while the other one would necessarily conserve it. Therefore, the inflationary (pre-big-bang ) epoch is born out of dilatonic matter violating the WEC. Recall that in the usual inflationary scenario the matter stress energy of the scalar field violates the Strong energy condition $\left(T_{\mu \nu} \xi^{\mu} \xi^{\nu}-\frac{1}{2} T g_{\mu \nu} \xi^{\mu} \xi^{\nu} \geq 0\right)$.

We now focus our attention on a more general class of metrics which are given as :

$$
d s^{2}=-d t^{2}+a_{1}^{2}(t) d x_{1}^{2}+a_{2}^{2}(t) d x_{2}^{2}+a_{3}^{2}(t) d x_{3}^{2}
$$

One can identify this class with the Kasner-type models (with power law choices for the scale factors), except that we do not necessarily impose any extra restrictions on the powers of $t$.

Note that our analysis can be easily extended to higher dimensions with essentially no modifications except for the presence of certain dimensionality factors.

The WEC inequalities for this case (from the L. H. S. of the Einstein equations) turn out to be given as :

$$
\begin{gathered}
\rho=\frac{\dot{a}_{1}}{a_{1}} \frac{\dot{a}_{2}}{a_{2}}+\frac{\dot{a}_{2}}{a_{2}} \frac{\dot{a}_{3}}{a_{3}}+\frac{\dot{a}_{3}}{a_{3}} \frac{\dot{a}_{1}}{a_{1}} \geq 0 \\
\rho+p_{1}=-\frac{\ddot{a}_{2}}{a_{2}}-\frac{\ddot{a}_{3}}{a_{3}}+\frac{\dot{a}_{1}}{a_{1}} \frac{\dot{a}_{2}}{a_{2}}+\frac{\dot{a}_{1}}{a_{1}} \frac{\dot{a}_{3}}{a_{3}} \geq 0 \\
\rho+p_{2}=-\frac{\ddot{a}_{1}}{a_{1}}-\frac{\ddot{a}_{3}}{a_{3}}+\frac{\dot{a}_{1}}{a_{1}} \frac{\dot{a}_{2}}{a_{2}}+\frac{\dot{a}_{2}}{a_{2}} \frac{\dot{a}_{3}}{a_{3}} \geq 0
\end{gathered}
$$




$$
\rho+p_{3}=-\frac{\ddot{a}_{1}}{a_{1}}-\frac{\ddot{a}_{2}}{a_{2}}+\frac{\dot{a}_{1}}{a_{1}} \frac{\dot{a}_{2}}{a_{2}}+\frac{\dot{a}_{2}}{2} \frac{\dot{a}_{3}}{a_{3}} \geq 0
$$

It is not entirely apparent from the above expressions whether the dual scale factors violate/satisfy the WEC or not. The fact is that we cannot make such a strong statement about all scale factors as we did in the case of the homogeneous isotropic model discussed previously.

However, the following restricted statement can be made. Assume pairs of dual scale factors which obey conditions : either $\dot{a}_{i}>0, \ddot{a}_{i}>0$ (inflationary regime) or $\dot{a}_{i}>0, \ddot{a}_{i}<0$ (FRW regime). Now it is clear from the inequalities that in the FRW regime the WEC must necessarily be satisfied whereas in the inflationary epoch it can to violated. More precisely, the second inequality, for example, implies that

$$
\frac{\ddot{a}_{2}}{a_{2}}+\frac{\ddot{a}_{3}}{a_{3}} \leq \frac{\dot{a}_{1}}{a_{1}}\left(\frac{\dot{a}_{2}}{a_{2}}+\frac{\dot{a}_{3}}{a_{3}}\right)
$$

In the FRW-like epoch ,the L.H.S. of the above inequality is always negative while the R.H.S. is necessarily positive - hence there is no way in which the inequality can be violated. On the other hand, in the inflationary era, violations of the WEC are possible although it is also true that it may be satisfied.

Let us now work out explicitly the inequalities for scale factors which obey a pole law in the inflationary epoch and a power (fractional or integral, but necessarily positive)law in the FRW -like regime.

Inflationary era :

$$
a_{1}(t)=(-t)^{-p} \quad ; \quad a_{2}(t)=(-t)^{-q} \quad ; \quad a_{3}(t)=(-t)^{-r} \quad t<0 \quad ; \quad 0<p, q, r<1
$$

FRW-like era :

$$
a_{1}(t)=t^{p} \quad ; \quad a_{2}(t)=t^{q} \quad ; \quad a_{3}(t)=t^{r} \quad t \geq 0 \quad ; \quad 0<p, q, r<1
$$

The WEC inequalities in the two epochs translate into the following :

Inflationary Era: 


$$
\begin{aligned}
p q+q r+r p & \geq 0 \\
-q^{2}-r^{2}+(q+r)(p-1) & \geq 0 \\
-p^{2}-r^{2}+(p+r)(q-1) & \geq 0 \\
-p^{2}-q^{2}+(p+q)(r-1) & \geq 0
\end{aligned}
$$

The first of these is trivially satisfied. The second, third and fourth imply :

$$
\begin{aligned}
& p \geq \frac{q^{2}+r^{2}}{q+r}+1 \\
& q \geq \frac{p^{2}+r^{2}}{p+r}+1 \\
& r \geq \frac{p^{2}+q^{2}}{p+q}+1
\end{aligned}
$$

Since $p, q, r$ are all less than 1 none of the above three can be satisfied.

FRW-like Era :

In this regime we have the following inequalities :

$$
\begin{aligned}
p q+q r+r p & \geq 0 \\
-q^{2}-r^{2}+(q+r)(p+1) & \geq 0 \\
-p^{2}-r^{2}+(p+r)(q+1) & \geq 0 \\
-p^{2}-q^{2}+(p+q)(r+1) & \geq 0
\end{aligned}
$$

The second, third and fourth inequalities therefore imply :

$$
\begin{aligned}
& p \geq \frac{q^{2}+r^{2}}{q+r}-1 \\
& q \geq \frac{p^{2}+r^{2}}{p+r}-1 \\
& r \geq \frac{p^{2}+r^{2}}{p+q}-1
\end{aligned}
$$

These are always satisfied, now because $p, q, r>0$.Therefore, we have demonstrated the existence of dual scale factors which necessarily satisfy/violate the WEC while being solutions to the field equations of the theory. 
Futhermore, one can ask whether the results stated above remain valid for general Brans-Dicke theories i.e. with the parameter $\omega$ not restricted to $\omega=-1$. Cosmological solutions as well as a generalisation of the scale factor duality symmetry has been found by Lidsey [21]. We briefly discuss the analogs of the above results now.

The SFD transformations now take the form :

$$
\begin{gathered}
\bar{a}=a^{\frac{2+3 \omega}{4+3 \omega}} \exp \left(-\frac{1+\omega}{4+3 \omega} \phi\right) \\
\bar{\phi}=-\frac{3}{4+3 \omega} \ln a-\frac{2+3 \omega}{4+3 \omega} \phi
\end{gathered}
$$

with $\omega \neq-\frac{4}{3}$.

As before, we obtain two branches related by SFD. The explicit solutions are :

$$
a^{ \pm}=t^{p_{ \pm}} \quad ; \quad e^{\phi}=t^{3 p_{ \pm}-1}
$$

where

$$
p_{ \pm}=\frac{1}{4+3 \omega}\left[1+\omega \pm\left(1+\frac{2 \omega}{3}\right)^{\frac{1}{2}}\right]
$$

It is easy to check that the WEC inequalities will be satisfied in both the + and branches (this depends on the sign of $p_{ \pm}$and therefore on the value of $\omega$, which we assume to be positive). Note that for $\omega=-1$ the duality between violation and conservation in the two branches appears. This is an additional check on our previous calculations.

Finally, let us discuss more general metrics which have off diagonal elements in their spatial part. We characterise such metrics by a generic form given as :

$$
d s^{2}=-d t^{2}+\mathcal{G}_{i k} \mathcal{G}_{k j} d x^{i} d x^{j}
$$

where $\mathcal{G}_{i j}$ is a $d \times d$ matrix. As before, one needs to write down the Energy Condition (WEC) inequalities and explore the consequences regarding their status. We shall confine ourselves to specific cases in $2+1$ dimensions.

Let us first consider the solution given in [22] for the equations of motion emerging from the low energy action with a term $V(\phi)$ containing the contribution of the dilaton potential 
as well as the cosmological constant. We have the following form for the line element and the dilaton field.

$$
\begin{gathered}
d s^{2}=-d t^{2}+g \tanh ^{2} t d x_{1}^{2}+\left(\psi+\frac{1}{4} g b^{2} \tanh ^{2} t\right) d x_{2}^{2}+b g \tanh ^{2} t d x_{1} d x_{2} \\
\phi=-\ln \cosh ^{2} t+\phi_{0}
\end{gathered}
$$

where $V=4$. A little algebra will reveal that the Einstein tensor $G_{\mu \nu}$ (in the one form basis $\left.d t, \sqrt{\psi} d x_{2}, \sqrt{g} \tanh t\left(d x_{1}+\frac{1}{2} b d x_{2}\right)\right)$ for this metric would turn out to be :

$$
G_{00}=G_{11}=G_{01}=G_{12}=0 \quad ; \quad G_{22}=2 \operatorname{sech}^{2} t \quad ;
$$

Therefore, it is clear that the WEC, which involves checking out the positivity of $G_{00}, G_{00}+$ $G_{i i} \quad(i=1,2)$, will be satisfied by this geometry.

Now, consider the dual geometry obtaining by acting on this metric by an $O(2,2)$ transformation. This is done by defining a matrix $M$ containing in general both the matrix $\mathcal{G}(t)$ (spatial part of the metric) and $\mathcal{B}(t)$ (the antisymmetric tensor potential) in the following combination.

$$
M=\left(\begin{array}{cc}
\mathcal{G}^{-1} & -\mathcal{G}^{-1} \mathcal{B} \\
-\mathcal{B G}^{-1} & \mathcal{G}-\mathcal{B G}^{-1} \mathcal{B}
\end{array}\right)
$$

The set of transformations which leave the action invariant are given as :

$$
M \rightarrow \Omega M \Omega^{T} \quad ; \quad \Phi=\phi+\ln \operatorname{det} G \rightarrow \Phi
$$

where $\Omega$ is an element of the $\mathrm{O}(\mathrm{d}, \mathrm{d})$ group $\left(\Omega \eta \Omega^{T}=\eta, \eta\right.$ is the Minkowski metric).

The solution given above is for $\mathcal{B}=0$. We now choose $\Omega$ to be :

$$
\Omega=\left(\begin{array}{cc}
\Pi & I-\Pi \\
I-\Pi & \Pi
\end{array}\right)
$$

with $\Pi^{2}=\Pi$. In $2+1$ dimensions, $\Pi$ can be chosen as :

$$
\Pi=\left(\begin{array}{ll}
0 & 0 \\
0 & 1
\end{array}\right)
$$


The $O(2,2)$ transformed version of the metric now becomes :

$$
d s^{2}=-d t^{2}+\frac{1}{g \tanh ^{2} t} d x_{1}^{2}+\psi d x_{2}^{2}
$$

The $G_{\mu \nu}$ for this metric is given as :

$$
G_{00}=G_{11}=G_{12}=G_{01}=0 \quad ; \quad G_{22}=-\frac{2}{\sinh ^{2} t}
$$

which clearly violates the WEC for all $t$ !

Alternatively, one can choose the matrix $\Pi$ to be :

$$
\Pi=\left(\begin{array}{ll}
1 & 0 \\
0 & 0
\end{array}\right)
$$

The $O(2,2)$ transformed metric now takes the form :

$$
d s^{2}=-d t^{2}+A^{2}(t)\left(g \psi \tanh ^{2} t d x_{1}^{2}+d x_{2}^{2}\right)
$$

where $A^{2}(t)=\frac{1}{\psi+\frac{1}{4} g b_{1}^{2} \tanh ^{2} t}$. Note that the $O(2,2)$ transformation generates a nontrivial torsion $\mathcal{B}$. We, however, do not need its explicit form in the discusion below. The WEC inequalities for this geometry are somewhat more involved. We require the following to hold true :

$$
\begin{gathered}
A^{4} \operatorname{sech}^{2} t\left[\tanh ^{2} t\left(\frac{1}{8} g^{2} b_{1}^{4}+\frac{3}{4} g b_{1}^{2} \psi\right)-\frac{1}{4} g b_{1}^{2} \psi\right] \leq 0 \\
A^{4} \operatorname{sech}^{2} t\left[-\frac{1}{4} g b_{1}^{2}\left(2+\operatorname{sech}^{2} t\right) \psi-2 \psi^{2}\right] \leq 0
\end{gathered}
$$

The question of whether the above inequalities hold good can only be answered by assuming the positivity/negativity of $\psi$ (other constants are taken as positive).

If $\psi$ is positive then the second inequality is trivially true whereas the first one would require $\psi \leq-\frac{1}{4} g b_{1}^{2}-$ which straightaway contradicts the assumption $\psi>0$. On the other hand, if $\psi$ is negative one can easily show that the Lorentzian signature of the metric is not retained for all $t$. 
Therefore, the $O(2,2)$ transformation yields a metric which violates the WEC - the choice of the value of $\psi$ does not play any role. Recall that the original metric (on which $O(2,2)$ was applied ) satisfied the WEC irrespective of the sign or values of the quantity $\psi$.

An extension to metrics in $3+1$ or higher dimensions can be achieved by following the algorithm given in [23]. The results concerning the status of the WEC are analogous to the $2+1$ dimensional case. We shall dwell upon this as well as many other cases in a future article [24].

To conclude, let us first summarize the results.

(i) The duality between conservation/violation of the WEC by dilatonic or other matter fields for spacetime geometries related by discrete SFD has been established. We have shown it for diagonal metrics which are isotropic as well as cases where anisotropy is present (Kasner like models). A brief statement about the continuation of these results to BD theories with $\omega \neq-1$ has also been made.

(ii) For the case of $O(d, d)$ related metrics we have outlined special cases in $2+1$ dimensions. A similar duality (though only through special cases) with regard to the violation/ conservation of the WEC is obtained for pairs of $O(d, d)$ related geometries.

It would be worth obtaining a general statement regarding this duality by studying the WEC inequalities for dual metrics related by $O(d, d)$. This would basically imply a classification of solutions in terms of their conservation/violation of the WEC or equivalently their features w.r.t. geodesic focusing. Such a phenomenon seems to be a feature of the low energy string effective actions in the string frame. Symmetries of the action and the equations of motion are (and should be) reflected on the behaviour and interplay of matter and geometry.

In the context of string cosmology, it is important to note that a pre-big-bang phase must necessarily be born out of matter violating the WEC. It would be instructive to check out the averaged energy conditions (AWEC, ANEC) [25] for this phase. Matter fields which generate a pre-big-bang phase could therefore be quantum in nature, considering the fact that quantum stress tensors can in principle violate the local energy conditions [26] but may 
satisfy their global (averaged) versions.

The author thanks M. Gasperini, A. Kumar, J. Maharana and T. Padmanabhan for useful discussions. Financial support from the Inter University Centre for Astronomy and Astrophysics, Pune, India is also gratefully acknowledged. 


\section{REFERENCES}

[1] G. Veneziano, Phys. Lett. B 265, 287 (1991)

[2] K. A. Meissner and G. Veneziano, Phys. Letts. B 267 , 33 (1991)

[3] K. A. Meissner and G. Veneziano, Mod. Phys. Letts. A 6, 3397 (1991)

[4] A. Sen, Phys. Letts. B 271, 295 (1991) ; Phys. Letts. B 274, 34 (1992) ; S. F. Hassan and A. Sen , Nucl. Phys. B 375, 103 (1991)

[5] J. Maharana and J. H. Schwarz, Nucl. Phys. B 390 , 3(1993)

[6] A. Sen, hep-th/9210050

[7] G. Horowitz, The dark side of string theory : black holes and black strings in String Theory and Quantum Gravity '92, ed. J. Harvey et.al., (World Scientific 1993).

[8] G. Veneziano, Status of string cosmology:basic concepts and main consequences in String gravity and physics at the Planck energy scale ed. N. Sanchez and A. Zichichi (Kluwer Academic Publishers, 1996)

[9] M. Gasperini, Status of string cosmology : phenomenological aspects in String gravity and physics at the Planck energy scale ed. N. Sanchez and A. Zichichi (Kluwer Academic Publishers , 1996)

[10] R. Brustein and G. Veneziano, Phys. Letts. B 329, 429 (1994); N. Kaloper, R. Madden and D. Olive, Nucl. Phys. B 452452 (1994);

[11] S. J. Rey, Phys. Rev. Letts. 77, 1929 (1996)

[12] C. Callan, S. B. Giddings, J. Harvey and A. Strominger, Phys. Rev. D45, 1005 (1992)

[13] M. Gasperini, J. Maharana and G. Veneziano, hep-th/9602087; J. Lidsey , gr-qc/9605127

[14] S. Kar, hep-th/9604047 
[15] R. Easther, K. Maeda and D. Wands, Phys. Rev. D 53, 4247 (1996) , R. Easther and K. Maeda, hep-th/9605173

[16] D. Welch, Phys. Rev. D 50, 6404 (1994)

[17] M. Gasperini, J. Maharana and G. Veneziano, Phys. Letts. B (1991)

[18] I. Antoniadis, J. Rizos and K. Tamvakis, Nucl. Phys. B 415, 497 (1994)

[19] R. M. Wald, General Relativity (University of Chicago Press, 1985)

[20] S. W. Hawking and G. F. R. Ellis, The large scale structure of spacetime (Cambridge University Press, 1972)

[21] J. Lidsey, hep-th/9609067

[22] S. P. Khastgir and A. Kumar, Mod. Phys. Letts. A6, 3365 (1991)

[23] S. K. Kar, S. P. Khastgir, A. Kumar, Mod. Phys. Letts. A7, 1542 (1992)

[24] S. Kar, (In preparation)

[25] F. Tipler, Phys. Rev. D 17, 2357 (1973) ; T. A. Roman, Phys. Rev. D 33, 2586 (1986) ibid. 37, 546 (1988); A. Borde, Class. Qtm. Grav. 4, 343 (1987)

[26] H. Epstein, V. Glaser and A. Yaffe, Nuovo Cimento 36, 2296 (1965) 